\title{
Transoral robotic surgery for oropharyngeal cancer: patient selection and special considerations
}

This article was published in the following Dove Press journal: Cancer Management and Research

\author{
R Michael Baskin' \\ Brian J Boyce ${ }^{1,2}$ \\ Robert Amdur ${ }^{2,3}$ \\ William M Mendenhall2,3 \\ Kathryn Hitchcock ${ }^{2,3}$ \\ Natalie Silver ${ }^{1,2}$ \\ Peter T Dziegielewski ${ }^{1,2}$ \\ 'Department of Otolaryngology, \\ University of Florida, Gainesville, FL, \\ USA; ${ }^{2}$ University of Florida Health \\ Cancer Center, Gainesville, FL, USA; \\ ${ }^{3}$ Department of Radiation Oncology, \\ University of Florida, Gainesville, FL, \\ USA
}

Correspondence: Peter T Dziegielewski Department of Otolaryngology, University of Florida, PO Box 100264 , I345 Center Dr., M2-228 MSB,

Gainesville, FL 32610, USA

Tel +l 3522735199

Fax +l 352392678 |

Email ptd@ufl.edu

\begin{abstract}
The increasing incidence of oropharyngeal squamous cell carcinoma (OPSCC) emphasizes the importance of optimizing treatment for the disease. Historical protocol has utilized definitive radiation and invasive open procedures; these techniques expose the patient to significant risks and morbidity. Transoral robotic surgery (TORS) has emerged as a therapeutic modality with promise. Here, the literature regarding proper patient selection and other considerations for this procedure was reviewed. Multiple patient and tumor-related factors were found to be relevant for successful use of this treatment strategy. Outcomes regarding early and advanced-stage OPSCC were analyzed. Finally, the literature regarding use of TORS in three distinct patient populations, individuals with primary OPSCC, carcinoma of unknown primary and those with recurrent OPSCC, was examined.
\end{abstract}

Keywords: oropharyngeal cancer, TORS, robotic surgery, carcinoma of unknown primary

\section{Introduction}

The incidence of oropharyngeal cancer (OPC) is steadily rising, with an estimated 17,000 new cases expected in the US in 2017. ${ }^{1}$ If current trends continue, the incidence of this malignancy will nearly double by the year $2030 .^{2}$ These increases are attributed to the surge in human papillomavirus (HPV) positive forms of oropharyngeal squamous cell carcinoma (OPSCC). From 1973 to 2004, the incidence of HPV-positive OPSCC increased by $225 \%$ and currently represents $~ 70 \%$ of newly diagnosed cases. ${ }^{2}$ Historically, OPC was treated with extensive surgery involving mandibular lingual release, mandibulotomy, and other maximally invasive techniques. These approaches resulted in complication rates ranging from 10 to $60 \%$ with the potential for long-term morbidity. ${ }^{3-5}$ In part due to the concern for these potential complications, primary surgery declined in popularity in favor of chemoradiation (CRT). Locoregional control and survival rates were similar to traditional surgery. From 1985 to 2001, the use of definitive CRT in the treatment of advanced OPC doubled, while the use of primary surgery significantly declined. ${ }^{6}$ However, the increased utilization of this modality led to recognition of its significant related long-term toxicities. Complications resulting from organ preservation therapies include mucositis, fibrosis, xerostomia, dermatitis, dysphagia, osteoradionecrosis, and neutropenia. ${ }^{78}$ Among these problems, dysphagia is one of the most frequent short- and long-term complications of CRT for OPSCC. Previous studies have demonstrated rates of severe late laryngopharyngeal toxicity, requiring gastrostomy tube nutrition, as high as $76 \%$ in OPC survivors. ${ }^{910}$ Therefore, 
both open surgical techniques and organ preservation therapy have the potential for multiple unfavorable side effects.

The long-term complications of OPC treatment have recently been heavily scrutinized. The vast majority of current OPSCC cases in North America and part of Europe are HPV-positive. These patients are often diagnosed at an earlier stage than their HPV-negative counterparts and have a much-improved overall prognosis. Life expectancy following treatment is increased, which in turn increases the window where negative sequela from cancer treatment can impact quality of life.

Transoral robotic surgery (TORS) was first developed by Dr Hockstein in 2005 with a case report of a supraglottic laryngectomy in a canine model. ${ }^{11}$ In the same year, the first case ofTORS used in vivo (ie, in human) was reported for a vallecular cyst. ${ }^{12}$ The technology underwent further development by Drs Weinstein and O'Malley at the University of Pennsylvania and was subsequently approved by the US Food and Drug Administration (FDA) in 2009 for use in head and neck surgery. ${ }^{11,13}$ The robot provides the following multiple technical advantages: improved visualization with three-dimensional imaging and angled scopes, tremor filter, and improved range of motion with robotic arms when compared to endoscopic and open techniques. ${ }^{13,14}$ These favorable attributes have resulted in excellent functional and oncologic outcomes in head and neck cancer patients. ${ }^{15-22}$ The results are in part due to the finding that utilization of TORS as first-line treatment may lead to a safe reduction in adjuvant therapy. ${ }^{23-26}$ However, the evidence for de-escalation is limited to retrospective studies, and prospective data are pending. TORS can be used with reduced post-operative radiation doses and often the omission of concurrent chemotherapy. ${ }^{16,17,27}$ It is well known that the majority of long-term functional impairment and worsened quality of life is directly related to open surgery and high-dose radiotherapy and/or concurrent chemotherapy. ${ }^{17}$ Moreover, in a climate of growing health care reform, TORS has been shown in multiple studies to potentially be more costeffective than primary CRT. ${ }^{11,18,28-30}$

While the advantages of TORS in the treatment of OPSCC are evident, appropriate patient selection is paramount. In this review, both patient and tumor-related factors that must be considered prior to implementation of TORS were identified. The literature related to robotic surgery in three OPSCC patient populations was discussed.

\section{Patient selections}

\section{Patient-related considerations}

The principles of minimally invasive head and neck surgical approaches, such as transoral laser microsurgery (TLM) and
TORS, are based on maximizing exposure while minimizing surgical morbidity. The majority of cases, if properly selected, should avoid tracheostomy, pharyngotomy, and formal flap reconstruction. Thus, healing is often accomplished by secondary intention. Although there are reports of local flaps (facial artery musculomucosa flap [FAMM], buccal fat, and palatal island) being used for reconstruction, these are unnecessary in most scenarios. Because of the open oropharyngeal wound, the post-operative course may involve bleeding, airway compromise, dehydration, and malnutrition. Operative candidates must be able to withstand these possible risks and be expected to recover and heal from the procedure. Comorbid conditions including immunosuppression, congestive heart failure, chronic obstructive lung disease (COPD), connective tissue or rheumatologic disease and conditions that prevent holding anticoagulation, poorly controlled diabetes, and malnutrition may all serve as strong relative or absolute contraindications for transoral surgeries in general and especially for TORS. ${ }^{31}$ In addition to patient comorbidities, details related to operative exposure must be addressed.

In 2009, Rich et al identified factors that required consideration for adequate endoscopic access in TLM. These criteria were referred to as the 8Ts of endoscopic access: teeth, trismus, transverse dimensions (mandibular), tori, tongue, tilt, treatment (prior radiation), and tumor. ${ }^{32}$ Since that publication these criteria have gained wide acceptance in patient selection for transoral endoscopic surgery. Many of these same principles are applicable to TORS. Trismus that prevents robotic access to the oral cavity is an obvious contraindication that has fit exclusion criteria in multiple studies. ${ }^{33-35}$ Limited neck extension that prohibits appropriate patient positioning is also recognized as a contraindication to TORS. ${ }^{36}$ Other groups have expanded the pre-operative considerations proposed by Rich et al to provide additional exclusion criteria related to TORS. Morbid obesity, micrognathia, microstomia, and craniofacial abnormalities have all been recognized as factors that may prevent robotic access. ${ }^{35,37,38}$ There is no agreed upon body mass index (BMI) that precludes the use of TORS, though for some a BMI of $>40$ serves as a relative contraindication. ${ }^{36}$

Many of the aforementioned contraindications, including trismus and micrognathia, are subjective physical examination findings that may be difficult to apply in the exclusion of TORS candidates. Therefore, various studies have sought to research specific anatomical characteristics and dimensions that represent ineligibility for TORS. Arora et $\mathrm{al}^{39}$ assessed 51 cadavers with no history of oropharyngeal disease or head and neck surgery. Their analysis characterized either 
"adequate" or "suboptimal" exposure of the base of tongue and epiglottis in each cadaver. They then recorded the following seven separate anthropometric measurements for each specimen: mandibular body length, mandibular body height, hyoid-mental distance, sterno-mental distance, thyro-mental distance, cricomental distance, and neck circumference. When comparing "adequate" to "suboptimal" robotic exposure of the base of tongue, it was found that the mean values for mandibular body height ( $2.6 \mathrm{~cm}$ versus $2.2 \mathrm{~cm}, p=0.03$ ), hyoid-mental distance ( $5.5 \mathrm{~cm}$ versus $4.8 \mathrm{~cm}, p=0.02)$, and neck circumference ( $38.7 \mathrm{~cm}$ versus $42.1 \mathrm{~cm}, p=0.04$ ) were statistically significant predictors. These three parameters were also statistically significant with regard to "adequate" exposure to the epiglottis. ${ }^{39}$

Luginbuhl et al utilized pre-operative imaging to determine anatomical characteristics that would be associated with sufficient robotic access in the base of tongue resections. Thirty-one patients who underwent attempted base of tongue resection with TORS were included and classified as providing either "adequate" or "restricted" exposure. Twenty cephalometric measurements were obtained from pre-operative computed tomographs (CTs) or magnetic resonance images (MRIs). Of the 20 parameters assessed, three were statistically significant in their association with restricted access: distance from posterior pharyngeal wall to hyoid ( $\leq 30 \mathrm{~mm}$ ); the angle between the epiglottis and vertical plain of the larynx $\left(\geq 130^{\circ}\right)$; and distance from the posterior pharyngeal wall to the soft palate $(\leq 8.1 \mathrm{~mm}) .{ }^{40}$

These studies demonstrate that contraindications to TORS may be recognized when obtaining a thorough patient history, completing a head and neck physical examination, or reviewing pre-operative imaging. Patient comorbidities must always be considered in treatment planning. However, as robotic techniques continue to evolve the anatomical characteristics that currently prove to be challenging may cease to cause difficulty in these procedures.

\section{Tumor-related considerations}

When assessing candidacy for TORS, or any transoral resection of OPC, the importance of reviewing the characteristics of each tumor cannot be understated. Attention must be paid to the exact location of the tumor and its involvement with the surrounding anatomical structures. Furthermore, the surgeon must extrapolate the extent of resection that will be required in order to obtain a negative margin. Weinstein et $\mathrm{al}^{31}$ identified three categories of contraindications related to tumor location or surrounding anatomy for a TORS resection relying on healing by secondary intention: vascular, functional, and oncologic.

\section{Vascular factors that would prohibit TORS include:}

1. Tonsillar malignancy with a retropharyngeal carotid artery.

2. Tumor at the midline of the tongue base or vallecula.

3. Tumor adjacent to the carotid bulb or internal carotid artery.

4. Encasement of the carotid artery by tumor or metastatic neck nodes.

Functional contraindications included:

1. Tumor resection requiring $\geq 50 \%$ of the deep tongue base musculature or posterior pharyngeal wall.

2. Resection of the tongue base and entire epiglottis.

Oncologic contraindications included patients with: ${ }^{18,31,34,38,41-43}$

1. T4b cancers

2. Unresectable neck disease

3. Multiple distant metastasis

4. Neoplastic-related trismus

5. Involvement of the prevertebral fascia

6. Involvement of the mandible or hyoid

7. Tumor extension into the soft tissues of the lateral neck

8. Eustachian tube involvement.

Some patients may fail to demonstrate the aforementioned contraindications but may not be optimal surgical candidates. Although a tumor may be resectable, the surgeon must consider the functional outcomes and surgical morbidity in treatment planning. The ideal candidates for TORS will benefit from the minimally invasive nature of the technique; and thus, the surgeon must be aware of situations where morbidity will not be minimized. Dziegielewski et al reviewed quality of life outcomes in 81 patients who underwent TORS for OPSCC. The research demonstrated that patients $\geq 55$ years old were nearly five times more likely to require a gastrostomy tube. Furthermore, TORS resection of more than one oropharyngeal subsite resulted in a 5.6-fold increased risk of gastrostomy tube insertion. ${ }^{42}$ Increased TNM stage is also associated with worsened swallowing outcomes. ${ }^{15,44}$ In addition to dysphagia, the potential for velopharyngeal insufficiency, nasopharyngeal reflux, speech difficulty, and tracheostomy must be considered depending on tumor location. These possible outcomes must then be discussed thoroughly with the patient before proceeding with surgery. 
In association with functional outcomes, the surgeon must also consider adjuvant therapies that will be indicated post-operatively. The goal should be for the utilization of TORS as first-line therapy to result in the reduction of adjuvant radiotherapy or CRT. However, various tumor characteristics may indicate that this is not possible. Zevallos et $\mathrm{al}^{45}$ performed a multivariate analysis of 514 patients who underwent endoscopic transoral surgery that included 369 TORS patients. Their cohort included individuals with T1-T4a OPSCC and N0-N2 disease. The results demonstrated that individuals with T2 tumor and those with N2 disease were significantly more likely to have positive margins. ${ }^{44}$ This pathologic outcome, along with others including extracapsular extension, may fail to reduce the recommended doses of adjuvant radiation and chemotherapy. These adjuvant therapies are well known to result in decreased quality of life scores. ${ }^{16,17,42,27}$ Failure to reduce adjuvant therapy is associated with not only worsened patient outcomes but also a decrease in cost-effectiveness. ${ }^{46}$ Therefore, it has been suggested that when extracapsular extension is suspected or surgical margins will likely be positive, patients may not be ideal candidates for TORS; primary CRT should be considered. ${ }^{47}$ In particular, tumors invading through the pharyngeal constrictor muscles into the parapharyngeal space will likely have positive margins. As trials progress, it may be possible to offer p16-positive OPCs less adjuvant treatment in these settings; however, data are still in the infant stage. ${ }^{48}$

\section{TORS for early-stage oropharyngeal cancers (TI-T2, N0-NI)}

While various patient and tumor-related factors must be considered prior to TORS, multiple studies demonstrate that this treatment modality has favorable oncologic outcomes in early-stage OPSCC. de Almeida et al reviewed 410 patients who underwent TORS. Within this cohort, $88.8 \%$ had OPC and $83.5 \%$ had T1-T2 disease. Their data demonstrated a 3 -year overall survival rate of $87.1 \%$ and a 3 -year diseasespecific survival rate of $94.5 \%{ }^{21}$ Multiple other reports have demonstrated similar results regarding TORS and adjuvant therapy as needed. These findings are equivalent or better than those of definitive radiation. ${ }^{20,22,24,48-50}$ Some authors have focused on TORS in those with HPV-negative earlystage OPSCC. Dabas et al reviewed 57 patients meeting this criteria and showed locoregional control in $95.8 \%$ and overall survival in $93.8 \%$ at a mean follow-up of 29 months. ${ }^{51}$ Other studies have corroborated these data by demonstrating a lack of significance of HPV status with regard to survival outcomes. $^{21,41}$ The efficacy of TORS as a sole treatment method has also been validated. Weinstein et al performed TORS as a single treatment modality in 30 patients with previously untreated OPSCC. A total of $70 \%$ of the patients enrolled in the study had T1-T2 primary tumors and N0-N1 nodal disease. At a minimum follow-up of 18 months, their data demonstrated a local control rate of $97 \%$ and an overall survival of $100 \% .{ }^{19}$ While TORS would ideally be utilized as the sole method of therapy, survival outcomes with TORS as a part of multimodality therapy have been high. ${ }^{52}$

\section{TORS for advanced-stage oropharyngeal cancers (T3-T4, N2-N3)}

The inclusion of surgery as a part of multimodality therapy for advanced OPSCC has been shown to be associated with improved survival. ${ }^{53}$ Most studies that include TORS involve tumors with low $\mathrm{T}$ stage but advanced cervical disease. In these cases, surgery with TORS is utilized as first-line therapy followed by adjuvant radiation and possibly chemotherapy. This literature demonstrates promising data regarding oncologic outcomes. White et al reviewed 89 patients including $65 \%$ that had either T3-T4 tumors or N2-N3 disease. A total of $92 \%$ of patients underwent surgery with TORS as first-line treatment for their OPSCC, and this cohort demonstrated $89.3 \%$ overall 2-year survival. ${ }^{49}$ Cohen et al completed a retrospective review that included 50 patients with $89.2 \%$ demonstrating stage III/IV OPSCC. Two-year overall survival for the entire cohort was $80.6 \%$ and disease-specific survival was $92.6 \%{ }^{41}$ Zenga et al examined survival outcomes of patients with T4 OPCs treated with transoral surgery versus primary CRT and also found a survival advantage with primary surgery. ${ }^{28}$ Those treated with non-surgical therapies had a 2-3-fold increased risk of death or disease recurrence. ${ }^{28}$ Aside from oncologic data, other benefits of TORS as first-line treatment include the ability for pathologic analysis and subsequent upstaging or downstaging of the patient's disease..$^{25}$ This may lead to reduction in doses of radiation and the possible avoidance of chemotherapy. ${ }^{26,54}$ Hurtuk et al reviewed 64 patients who underwent TORS including 68.4\% with N2-N3 classification. The ability to review pathologic specimens resulted in avoidance of chemotherapy in $34 \%$ of those with stage III/ IV tumors. ${ }^{55}$ Functional and quality of life outcomes demonstrated significant improvements.

\section{Special considerations Unknown primary}

It is estimated that cervical metastasis from an unknown primary site represents $2-4 \%$ of all squamous cell carcinomas 
of the head and neck. ${ }^{56}$ Localization of a primary tumor has been reported to increase survival rates while decreasing radiotherapy doses and fields and its negative sequelae..$^{26,57-59}$ The typical workup in patients with a carcinoma of unknown primary (CUP) includes thorough history and physical examinations, flexible nasolaryngoscopy, and imaging studies including CT, MRI, and/or positron-emission tomography/ computed tomography (PET/CT). If these modalities have failed to localize the primary tumor, panendoscopy with or without tonsillectomy and directed biopsies of nasopharynx, oropharynx, and larynx are typically completed. However, $40-50 \%$ of primary tumors remain unknown following these measures. ${ }^{60,61}$ Given that the majority of these tumors are suspected to be in the base of tongue or palatine tonsil, TORS is an excellent diagnostic modality in this population. Multiple studies ${ }^{56-67}$ have reported on the use of TORS in CUP patients. In these reports, robotic surgery may be performed following a negative panendoscopy with benign directed biopsies. TORS is utilized to perform palatine tonsillectomy and lingual tonsillectomy. Whether these procedures are unilateral or bilateral depends upon the protocol of each facility. Completion of bilateral surgery is supported by multiple studies demonstrating that malignancy in the contralateral lingual or palatine tonsil is found in $\sim 10 \%$ of cases. ${ }^{62-64}$ Some groups also choose to perform radical ipsilateral palatine tonsillectomy, while others do not include the superior pharyngeal constrictor muscle. Still others have developed TORS protocols for CUP which include neck dissection. ${ }^{63,64}$ The addition of tonsillectomy in these cases has shown to increase survival by $20 \% .53,54$ Overall, the robotic approach has resulted in identification of the primary site in $67-90 \%$ of patients. ${ }^{26,59,63,67,68}$ In addition to its use as a diagnostic tool, TORS may also serve a therapeutic role. The robotic approach has resulted in negative margins of these "found" primary tumors in $51-62 \%$ of cases. ${ }^{26,59,63}$ This pathologic outcome results in a safe reduction in adjuvant therapy and its potential complications. ${ }^{65}$ These benefits have resulted in data that support TORS as a cost-effective treatment in this patient population. ${ }^{69}$ The long-term survival benefits of this approach are yet to be elucidated, but current evidence suggests that patients will benefit.

\section{Surgical salvage}

For the past few decades, the preferred modality of treatment for OPSCC was radiation \pm chemotherapy. Therefore, for the majority of patients whose disease recurs, radiation is no longer an option, and surgery is the only therapy that may be used with curative intent. Open procedures in salvage patients often necessitate mandibulotomy and/or pharyngotomy, free flap reconstruction, tracheostomy, and additional procedures. These can be wrought with potential complications including poor wound healing, fistula, nonunion, bone exposure, and hardware extrusion. Previous studies have reported complication rates of $\sim 50 \%$ following these procedures in salvage patients. ${ }^{4}$ Oncologic outcomes are poor with 5-year disease-free survival rates $\sim 20 \% .{ }^{60-71}$ The robotic approach may provide a viable alternative. Few studies have reviewed outcomes of TORS in salvage patients; however, the limited data are thus far encouraging. White et al reported on a cohort of 128 patients evenly divided between salvage TORS and salvage open approaches. The two groups were matched with regard to patient demographics and tumor characteristics. TORS was associated with significant reduction in tracheostomy, gastric tube dependence, hospital stay, blood loss, operative time, and positive margins. Furthermore, the 2-year disease-free survival in the TORS patients was significantly higher than the open group ( $74 \%$ versus $43 \%, p=0.01) .{ }^{34}$ Data reported by Meulemans et al ${ }^{72}$ demonstrated similar findings with a 2 -year disease-free survival of $75.8 \%$. Dean et al reported functional outcomes comparing TORS for primary neoplasms, TORS as salvage surgery for recurrent disease, and open surgery for recurrent tumor. Their data showed that $43 \%$ of patients in the open group were gastrostomy tube dependent at 6 months, while $0 \%$ of patients in both robotic groups fit this description. ${ }^{43}$ These studies demonstrate that the benefit of TORS in the salvage setting is improved perioperative and functional outcomes of TORS in comparison to open procedures in salvage patients. Moreover, there is a potential for improved oncologic outcomes with improved access to the tumor via TORS and the possibility of adjuvant treatment in selected patients. Further study is warranted to validate these findings and clarify which patients are most appropriate for this treatment modality.

\section{Future directions}

Numerous questions remain regarding the application of TORS in the treatment of OPC. Debate persists regarding the definition of a "close margin" and the pathologic indications for adjuvant therapy. ${ }^{73,74}$ Prospective trials focusing on margin status and subsequent survival outcomes will identify patients who may avoid high levels of adjuvant treatment. Furthermore, these data may be utilized to validate omission of the primary site to the field treated with post-operative radiation. ${ }^{75,76}$ These reductions in adjuvant therapy would be expected to improve functional outcomes and decrease overall cost. TORS utilization for CUP continues to evolve. 
Discrepancies exist among institutions regarding the extent of surgery required for these cases. Further research may elucidate whether surgery with TORS should be used to complete bilateral palatine and lingual tonsillectomy. It may also determine whether radical tonsillectomy should be completed and answer the question of bilateral versus unilateral neck dissection. Further research is needed regarding appropriate patient selection in salvage cases.

Due to the known long-term toxicities of CRT, several ongoing clinical trials are focused on determining if treatment may be de-escalated. The Adjuvant De-escalation, Extracapsular Spread, p16 Positive, Transoral (ADEPT) trial (unpublished data) was launched in 2013 to determine if chemotherapy may be omitted from high-risk OPC patients who are HPV positive and treated with transoral surgery. The ECOG 3311 trial is another ongoing example where low-risk HPV-positive OPCs (T1-T2, N0-N1) are treated with transoral surgery and no adjuvant radiation therapy or a low dose of adjuvant RT. ${ }^{37}$ Thus far, $39 \%$ of patients in the trial have been able to receive deintensified treatment safely. The Oropharynx: Radiotherapy Versus Trans-Oral Robotic Surgery (ORATOR) trial is a single-institution trial comparing quality of life and survival outcomes in OPCs treated with transoral surgery or primary RT. ${ }^{77}$ The Post-operative Adjuvant Treatment for HPV-positive Tumours (PATHOS) trial hopes to identify patients in whom adjuvant treatment can be deintensified after transoral surgery. The trial schema randomizes patients based on adverse pathological features to either 60 Gy or 50 Gy adjuvant RT with or without chemotherapy. ${ }^{78}$ It is only through trials like these that the true role of TORS and deintensified treatment in OPC will become elucidated.

Finally, the new pathologic staging regarding HPVpositive OPSCC must be considered. Studies utilizing TORS must be completed with the application of this staging paradigm in order to identify appropriate patient selection as well as report oncologic and functional outcomes.

\section{Conclusion}

While health care continues to battle the competing forces of improved care and reduced costs, TORS may be able to achieve these two conflicting goals. This treatment modality has demonstrated favorable oncologic and functional outcomes while reducing costs by decreasing adjuvant therapy and its associated morbidity. However, to reap these benefits the surgeon must exercise appropriate patient selection. This practice necessitates consideration of multiple patient and tumor-related characteristics. When these principles are applied, TORS has shown efficacy with both early and advanced-stage OPSCC. This technique may also play a role in improving the treatment of those with CUP and recurrent disease. Indeed, TORS has proven itself as a viable modality in the treatment of OPSCC.

\section{Disclosure}

The authors report no conflicts of interest in this work.

\section{References}

1. Siegel R, Miller K, Jemal A. Cancer statistics, 2017. CA Cancer J Clin. 2017;67(1):7-30.

2. Chaturvedi A, Engels E, Pfeiffer R, et al. Human Papillomavirus and rising oropharyngeal cancer incidence in the United States. J Clin Oncol. 2011;29(32):4294-4301.

3. Parsons J, Mendenhall W, Stringer S, et al. Squamous cell carcinoma of the oropharynx: surgery, radiation therapy, or both. Cancer. 2002;94(11):2967-2980.

4. Zafereo ME, Weber RS, Lewin JS, Roberts DB, Hanasono MM. Complications and functional outcomes following complex oropharyngeal reconstruction. Head Neck. 2010;32(8):1003-1011.

5. Dziegielewski PT, Mlynarek AM, Dimitry J, Harris JR, Seikaly H. The mandibulotomy: friend or foe? Safety outcomes and literature review. Laryngoscope. 2009;119(12):2369-2375.

6. Chen AY, Schrag N, Hao Y, et al. Changes in the treatment of advanced oropharyngeal cancer, 1985-2001. Laryngoscope. 2007;117(1):16-21.

7. Yeh DH, Tam S, Fung K, et al. Transoral robotic surgery vs. radiotherapy for management of oropharyngeal squamous cell carcinoma - a systemic review of the literature. Eur J Surg Oncol. 2015;41(12):1603-1614.

8. Byrd JK, Ferris RL. Is there a role for robotic surgery in the treatment of head and neck cancer? Curr Treat Options Oncol. 2016;17(6):29.

9. Machtay M, Moughan J, Trotti A, et al. Factors associated with severe late toxicity after concurrent chemoradiation for locally advanced head and neck cancer: an RTOG analysis. J Clin Oncol. 2008;26(21):3582-3589.

10. Hutcheson KA, Lewin JS, Barringer DA, et al. Late dysphagia after radiotherapy-based treatment of head and neck cancer. Cancer. 2012;118(23):5793-5799.

11. Weinstein GS, O’Malley BW, Hockstein NG. Transoral robotic surgery: supraglottic laryngectomy in a canine model. Laryngoscope. 2005;115(7):1315-1319.

12. McLeod IK, Melder PC. Da Vinci robot-assisted excision of a vallecular cyst: a case report. Ear Nose Throat J. 2005;84(3):170-172.

13. Cracchiolo JR, Roman BR, Kutler DI, Kuhel WI, Cohen MA. Adoption of transoral robotic surgery compared with other surgical modalities for treatment of oropharyngeal squamous cell carcinoma. J Surg Oncol. 2016;114(4):405-411.

14. Duek I, Billan S, Amit M, Gil Z. Transoral robotic surgery in the HPV era. Rambam Maimonides Med J. 2014;5(2):e0010.

15. Hutcheson KA, Holsinger FC, Kupferman ME, et al. Functional outcomes after TORS for oropharyngeal cancer: a systematic review. Eur Arch Otorhinolaryngol. 2015;272(2):463-471.

16. Leonhardt FD, Quon H, Abrahão M, O’Malley BW Jr, Weinstein GS. Transoral robotic surgery for oropharyngeal carcinoma and its impact on patient-reported quality of life and function. Head Neck. 2012;32(2):146-154.

17. Hurtuk AM, Marcinow A, Agrawal A, Old M, Teknos TN, Ozer E. Quality-of-life outcomes in transoral robotic surgery. Otolaryngol Head Neck Surg. 2012;146(1):68-73.

18. Moore EJ, Hinni ML, Olsen KD, Price DL, Laborde RR, Inman JC. Cost considerations in the treatment of oropharyngeal squamous cell carcinoma. Otolaryngol Head Neck Surg. 2012;146(6):946-951.

19. Weinstein GS, Quon H, Newman HJ, et al. Transoral robotic surgery alone for oropharyngeal cancer: an analysis of local control. Arch Otolaryngol Head Neck Surg. 2012;138(7):628-634. 
20. van Loon JW, Smeele LE, Hilgers FJ, van den Brekel MW. Outcome of transoral robotic surgery for stage I-II oropharyngeal cancer. Eur Arch Otorhinolaryngol. 2015;272(1):175-183.

21. de Almeida JR, Li R, Magnuson JS, et al. Oncologic outcomes after transoral robotic surgery: a multi-institutional study. JAMA Otolaryngol Head Neck Surg. 2015;141(12):1043-1051.

22. Morisod B, Simon C. Meta-analysis on survival of patients treated with transoral surgery versus radiotherapy for early-stage squamous cell carcinoma of the oropharynx. Head Neck. 2016;38(suppl 1):E2143-E2150.

23. Motz K, Chang HY, Quon H, et al. Association of transoral robotic surgery with short-term and long-term outcomes and costs of care in oropharyngeal cancer surgery. JAMA Otolaryngol Head Neck Surg. 2017;143(6):580-588.

24. Genden EM, Kotz T, Tong CC, et al. Transoral robotic resection and reconstruction for head and neck cancer. Laryngoscope. 2011;121(8): 1668-1674.

25. Smith RV, Schiff BA, Garg M, Haigentz M. The impact of transoral robotic surgery on the overall treatment of oropharyngeal cancer patients. Laryngoscope. 2015;125(suppl 10):S1-S15.

26. Patel SA, Magnuson JS, Holsinger FC, et al. Robotic surgery for primary head and neck squamous cell carcinoma of unknown site. JAMA Otolaryngol Head Neck Surg. 2013;139(11):1203-1211.

27. Levendag PC, Teguh DN, Voet P, et al. Dysphagia disorders in patients with cancer of the oropharynx are significantly affected by the radiation therapy dose to the superior and middle constrictor muscle: a dose-effect relationship. Radiother Oncol. 2007;85(1):64-73.

28. Zenga J, Wilson M, Adkins DR, et al. Treatment outcomes for T4 Oropharyngeal squamous cell carcinoma. JAMA Otolaryngol Head Neck Surg. 2015;141(12):1118-1127.

29. Richmon JD, Quon H, Gourin CG. The effect of transoral robotic surgery on short-term outcomes and cost of care after oropharyngeal cancer surgery. Laryngoscope. 2014;124(1):165-171.

30. de Almeida JR, Byrd JK, Wu R, et al. A systematic review of transoral robotic surgery and radiotherapy for early oropharynx cancer: a systematic review. Laryngoscope. 2014;124(9):2096-2102.

31. Weinstein GS, O’Malley BW, Rinaldo A, et al. Understanding contraindications for transoral robotic surgery (TORS) for oropharyngeal cancer. Eur Arch Otorhinolaryngol. 2015;272(7):1551-1552.

32. Rich JT, Milov S, Lewis JS, et al. Transoral laser microsurgery (TLM) \pm adjuvant therapy for advanced stage oropharyngeal cancer: outcomes and prognostic factors. Laryngoscope. 2009;119(9):1709-1719.

33. Moore EJ, Olsen KD, Kasperbauer JL. Transoral robotic surgery for oropharyngeal squamous cell carcinoma: a prospective study of feasibility and functional outcomes. Laryngoscope. 2009;119(11):2156-2164

34. White H, Ford S, Bush B, et al. Salvage surgery for recurrent cancers of the oropharynx: comparing TORS with standard open surgical approaches. JAMA Otolaryngol Head Neck Surg. 2013;139(8): 773-778.

35. Ohba S, Fujimaki M, Kojima M, et al. Transoral robotic surgery for head and neck cancer: a review. Juntendo Med J. 2015;61(1):21-24.

36. Vasan NR. Robotic Surgery in Otolaryngology Head and Neck Surgery. London, United Kingdom: JP Medial Ltd; 2015.

37. Holsinger FC, Ferris RL. Trans oral endoscopic head and neck surgery and its role within the multidisciplinary treatment paradigm of oropharynx cancer: robotics, lasers, and clinical trials. J Clin Oncol. 2015;33(29):3285-3292.

38. Loevner LA, Learned KO, Mohan S, et al. Transoral robotic surgery in head and neck cancer: what radiologists need to know about the cutting edge. Radiographics. 2013;33(6):1759-1779.

39. Arora A, Kotecha J, Acharya A, et al. Determination of biometric measures to evaluate patient suitability for transoral robotic surgery. Head Neck. 2015;37(9):1254-1260.

40. Luginbuhl A, Baker A, Curry J, Drejet S, Miller M, Cognetti D. Preoperative cephalometric analysis to predict transoral robotic surgery exposure. J Robot Surg. 2014;8(4):313-317.

41. Cohen MA, Weinstein GS, O’Malley BW Jr, Feldman M, Quon H. Transoral robotic surgery and human papillomavirus status: oncologic results. Head Neck. 2011;33(4):573-580.
42. Dziegielewski PT, Teknos TN, Durmus K, et al. Transoral robotic surgery for oropharyngeal cancer: long-term quality of life and functional outcomes. JAMA Otolaryngol Head Neck Surg. 2013;139(11): 1099-1108.

43. Dean NR, Rosenthal EL, Carroll WR, et al. Robotic-assisted surgery for primary or recurrent oropharyngeal carcinoma. Arch Otolaryngol Head Neck Surg. 2010;136(4):380-384.

44. More YI, Tsue TT, Girod DA, et al. Functional swallowing outcomes following transoral robotic surgery vs primary chemoradiotherapy in patients with advanced-stage oropharynx and supraglottic cancers. JAMA Otolaryngol Head Neck Surg. 2013;139(1):43-48.

45. Zevallos JP, Mitra N, Swisher-McClure S. Patterns of care and perioperative outcomes in transoral endoscopic surgery for oropharyngeal squamous cell carcinoma. Head Neck. 2016;38(3):402-409.

46. Rodin D, Caulley L, Burger E, et al. Cost-effectiveness analysis of radiation therapy versus transoral robotic surgery for oropharyngeal squamous cell carcinoma. Int J Radiat Oncol Biol Phys. 2017; 97(4):709-717.

47. de Almeida JR, Moskowitz AJ, Miles BA, et al. Cost-effectiveness of transoral robotic surgery versus (chemo)radiotherapy for early $\mathrm{T}$ classification oropharyngeal carcinoma: a cost-utility analysis. Head Neck. 2016;38(4):589-600.

48. An Y, Park HS, Kelly JR, et al. The prognostic value of extranodal extension in human papillomavirus-associated oropharyngeal squamous cell carcinoma. Cancer. 2017;123(14):2762-2772.

49. White HN, Moore EJ, Rosenthal EL, et al. Transoral robotic-assisted surgery for head and neck squamous cell carcinoma: one- and 2-year survival analysis. Arch Otolaryngol Head Neck Surg. 2010;136(12):1248-1252.

50. Ford SE, Brandwein-Gensler M, Carroll WR, Rosenthal EL, Magnuson JS. Transoral robotic versus open surgical approaches to oropharyngeal squamous cell carcinoma by human papillomavirus status. Otolaryngol Head Neck Surg. 2014;151(4):606-611.

51. Dabas S, Gupta K, Ranjan R, Sharma AK, Shukla H, Dinesh A. Oncological outcome following de-intensification of treatment for stage I and II HPV negative oropharyngeal cancers with transoral robotic surgery (TORS): a prospective trial. Oral Oncol. 2017;69:80-83.

52. Roden DF, Schreiber D, Givi B. Multimodality treatment for early-stage tonsil cancer. Otolaryngol Head Neck Surg. 2017;157(1):62-68.

53. Roden DF, Schreiber D, Givi B. Triple-modality treatment in patients with advanced stage tonsil cancer. Cancer. 2017;123(17):3269-3276.

54. Haughey BH, Hinni ML, Salassa JR, et al. Transoral laser microsurgery as primary treatment for advanced-stage oropharyngeal cancer: a United States multicenter study. Head Neck. 2011;33(12):1683-1694.

55. Hurtuk A, Agrawal A, Old M, Teknos TN, Ozer E. Outcomes of transoral robotic surgery: a preliminary clinical experience. Otolaryngol Head Neck Surg. 2011;145(2):248-253.

56. Waltonen JD, Ozer E, Hall NC, Schuller DE, Agrawal A. Metastatic carcinoma of the neck of unknown primary origin: evolution and efficacy of the modern workup. Arch Otolaryngol Head Neck Surg. 2009;135(10):1024-1029.

57. Haas I, Hoffmann TK, Engers R, Ganzer U. Diagnostic strategies in cervical carcinoma of an unknown primary (CUP). Eur Arch Otorhinolaryngol. 2002;259(6):325-333.

58. Davis KS, Byrd JK, Mehta V, et al. Occult primary head and neck squamous cell carcinoma: utility of discovering primary lesions. Otolaryngol Head Neck Surg. 2014;151(2):272-278.

59. Fu TS, Foreman A, Goldstein DP, de Almeida JR. The role of transoral robotic surgery, transoral laser microsurgery, and lingual tonsillectomy in the identification of head and neck squamous cell carcinoma of unknown primary origin: a systematic review. J Otolaryngol Head Neck Surg. 2016;45(1):28.

60. Cianchetti M, Mancuso AA, Amdur RJ, et al. Diagnostic evaluation of squamous cell carcinoma metastatic to cervical lymph nodes from an unknown head and neck primary site. Laryngoscope. 2009;119(12):2348-2354.

61. Pattani KM, Goodier M, Lilien D, Kupferman T, Caldito G, Nathan CO. Utility of panendoscopy for the detection of unknown primary head and neck cancer in patients with a negative PET/CT scan. Ear Nose Throat J. 2011;90(8):E16-E20. 
62. Koch WM, Bhatti N, Williams MF, Eisele DW. Oncologic rationale for bilateral tonsillectomy in head and neck squamous cell carcinoma of unknown primary source. Otolaryngol Head Neck Surg. 2001;124(3):331-333.

63. Geltzeiler M, Doerfler S, Turner M, et al. Transoral robotic surgery for management of cervical unknown primary squamous cell carcinoma: updates on efficacy, surgical technique, and margin status. Oral Oncol. 2017;66:9-13.

64. Dziegielewski PT, Boyce BJ, Old M, et al. Transoral robotic surgery for tonsillar cancer: addressing the contralateral tonsil. Head Neck. 2017;39(11):2224-2231.

65. Kang SY, Dziegielewski PT, Old MO, Ozer E. Transoral robotic surgery for carcinoma of unknown primary in the head and neck. J Surg Oncol. 2015;112(7):697-701.

66. Durmus K, Rangarajan SV, Old MO, Agrawal A, Teknos TN, Ozer E. Transoral robotic approach to carcinoma of unknown primary. Head Neck. 2014;36(6):848-852.

67. Mehta V, Johnson P, Tassler A, et al. A new paradigm for the diagnosis and management of unknown primary tumors of the head and neck: a role for transoral robotic surgery. Laryngoscope. 2013;123(1): 146-151.

68. Patel SA, Parathaneni A, Parvathaneni U, et al. Post-operative therapy following transoral robotic surgery for unknown primary cancers of the head and neck. Oral Oncol. 2017;72:150-156.

69. Byrd JK, Smith KJ, de Almeida JR, et al. Transoral robotic surgery and the unknown primary: a cost-effectiveness analysis. Otolaryngol Head Neck Surg. 2014;150(6):976-982.

70. Patel SN, Cohen MA, Givi B, et al. Salvage surgery for locally recurrent oropharyngeal cancer. Head Neck. 2016;38(Suppl 1):E658-E664.
71. Righini CA, Nadour K, Faure C, et al. Salvage surgery after radiotherapy for oropharyngeal cancer. Treatment complications and oncological results. Eur Ann Otorhinolaryngol Head Neck Dis. 2012;129(1):11-16.

72. Meulemans J, Vanclooster C, Vauterin T, et al. Up-front and salvage transoral robotic surgery for head and neck cancer: a Belgian multicenter retrospective case series. Front Oncol. 2017;7:15.

73. Rubek N, Channir HI, Charabi BW, et al. Primary transoral robotic surgery with concurrent neck dissection for early stage oropharyngeal squamous cell carcinoma implemented at a Danish head and neck center: a phase II trial on feasibility and tumour margin status. Eur Arch Otorhinolaryngol. 2017;274(5):2229-2237.

74. Morisod B, Venara-Vulpe II, Alzuphar S, et al. Minimizing adjuvant treatment after transoral robotic surgery through surgical margin revision and exclusion of radiographic extracapsular extension: a prospective observational cohort study. Head Neck. 2017;39(5):965-973.

75. Ward MC, Kovfman SA. Transoral robotic surgery: the radiation oncologist's perspective. Oral Oncol. 2016;60:96-102.

76. Ojerholm E, Lukens JN, Ahn PH, et al. Benefits of omitting primary site radiation therapy after transoral robotic surgery: only time will tell. Pract Radiat Oncol. 2017;7(3):e157-e158.

77. Nichols AC, Yoo J, Hammond JA, et al. Early-stage squamous cell carcinoma of the oropharynx: radiotherapy vs. trans-oral robotic surgery (ORATOR) - study protocol for a randomized phase II trial. BMC Cancer. 2013;13:133.

78. Owadally W, Hurt C, Timmins H, et al. PATHOS: a phase II/III trial of risk-stratified, reduced intensity adjuvant treatment in patients undergoing transoral surgery for Human papillomavirus (HPV) positive oropharyngeal cancer. BMC Cancer. 2015; 15:602.
Cancer Management and Research

\section{Publish your work in this journal}

Cancer Management and Research is an international, peer-reviewed open access journal focusing on cancer research and the optimal use of preventative and integrated treatment interventions to achieve improved outcomes, enhanced survival and quality of life for the cancer patient. The manuscript management system is completely online and includes

\section{Dovepress}

a very quick and fair peer-review system, which is all easy to use. Visit http://www.dovepress.com/testimonials.php to read real quotes from published authors. 